\title{
Note: design and construction of a multi-scale, high-resolution, tube-generated x-ray computed-tomography system for three-dimensional (3D) imaging
}

\author{
Mertens, J C E ; Williams, J J ; Chawla, Nikhilesh
}

\begin{abstract}
The design and construction of a high resolution modular x-ray computed tomography (XCT) system is described. The approach for meeting a specified set of performance goals tailored toward experimental versatility is highlighted. The instrument is unique in its detector and x-ray source configuration, both of which enable elevated optimization of spatial and temporal resolution. The process for component selection is provided. The selected components are specified, the custom component design discussed, and the integration of both into a fully functional XCT instrument is outlined. The novelty of this design is a new lab-scale detector and imaging optimization through x-ray source and detector modularity.
\end{abstract}

DOI: https://doi.org/10.1063/1.4861924

Posted at the Zurich Open Repository and Archive, University of Zurich

ZORA URL: https://doi.org/10.5167/uzh-136162

Journal Article

Published Version

Originally published at:

Mertens, J C E; Williams, J J; Chawla, Nikhilesh (2014). Note: design and construction of a multi-scale, high-resolution, tube-generated x-ray computed-tomography system for three-dimensional (3D) imaging. Review of Scientific Instruments, 85(1):016103.

DOI: https://doi.org/10.1063/1.4861924 
Note: Design and construction of a multi-scale, high-resolution, tube-generated X-Ray computed-tomography system for three-dimensional (3D) imaging

J. C. E. Mertens, J. J. Williams, and Nikhilesh Chawla

Citation: Review of Scientific Instruments 85, 016103 (2014); doi: 10.1063/1.4861924

View online: http://dx.doi.org/10.1063/1.4861924

View Table of Contents: http://aip.scitation.org/toc/rsi/85/1

Published by the American Institute of Physics

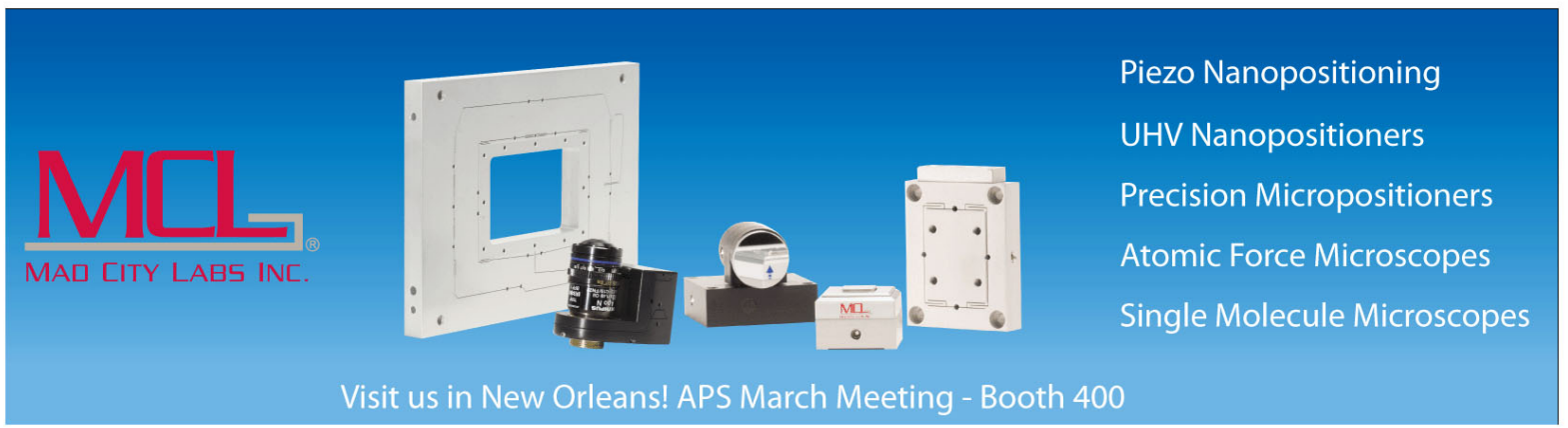




\title{
Note: Design and construction of a multi-scale, high-resolution, tube-generated X-Ray computed-tomography system for three-dimensional (3D) imaging
}

\author{
J. C. E. Mertens, J. J. Williams, and Nikhilesh Chawla \\ Materials Science and Engineering, Security and Defense Systems Initiative, Arizona State University, \\ 781 E. Terrace Road, ISTB4, Tempe, Arizona 85287-5604, USA
}

(Received 2 July 2013; accepted 30 December 2013; published online 14 January 2014)

\begin{abstract}
The design and construction of a high resolution modular x-ray computed tomography (XCT) system is described. The approach for meeting a specified set of performance goals tailored toward experimental versatility is highlighted. The instrument is unique in its detector and x-ray source configuration, both of which enable elevated optimization of spatial and temporal resolution. The process for component selection is provided. The selected components are specified, the custom component design discussed, and the integration of both into a fully functional XCT instrument is outlined. The novelty of this design is a new lab-scale detector and imaging optimization through $\mathrm{x}$-ray source and detector modularity. (O) 2014 AIP Publishing LLC. [http://dx.doi.org/10.1063/1.4861924]
\end{abstract}

Select research facilities employ synchrotron light sources in x-ray computed tomography (XCT) experiments (ex. Beamline 2BM, Advanced Photon Source, Argonne National Laboratories); these facilities are expensive, limited in number, and in high demand, implying short experiments at low frequencies. Lab-scale devices (Xradia, Inc., CA, Bruker Corporation, WI) have been commercially developed for quality control and even academic research, allowing XCT in the absence of a synchrotron facility.

Previous researchers ${ }^{1-6}$ have shown the potential of custom detectors and full lab-scale XCT instruments, providing insight to aspects of the design. Those that have developed detectors focus on synchrotron XCT, ${ }^{4,5}$ and those which apply to lab-scale adopt direct X-ray detectors or fiber-optic scintillator-chip coupled detectors. ${ }^{2}$ Those which have focused on detector optimization for lab-scale system applications merely survey commercially available detectors. ${ }^{3,6} \mathrm{~A}$ design for optimizing detection efficiency of lab-scale systems is needed. The current instrument's design provides a consolidation of these principles to yield a new, truly optimized, and modular lab-scale x-ray microtomography system.

The needs of a lab-scale x-ray detector, opposed to synchrotron XCT detectors, arise due to the differences in the X-ray beam used for imaging. Synchrotron XCT uses a relatively low-energy, monochromatic, parallel $\mathrm{x}$-ray beam, whereas cathode-tube derived braking $\mathrm{x}$-radiation is polychromatic and emitted in a diverging conic beam and can be relatively much higher in energy. The energy of the $\mathrm{x}$-rays that are to be detected dictates the choice of x-ray detector, due to band-edges in the phosphor and a dependence on $\mathrm{x}$-ray absorption with X-ray energy. In synchrotron XCT, it is critical to achieve submicron resolution at the scintillator crystal itself, because no x-ray magnification is achieved with the parallel beam. In cathode-tube derived $x$-rays, a cone-beam of emission is achieved providing $\mathrm{x}$-ray magnification; this magnification allows submicron features to be magnified onto the detector, relaxing the constraints on the imaging resolution of the phosphor itself.

The justification for the design and construction of a custom system (Fig. 1) is provided by the performance goals established for a desirable instrument:

- Spatial resolution of less than $1 \mu \mathrm{m}$

- Imaging of specimens of up to $10 \mathrm{~mm}$ in diameter

- Imaging of high atomic number sample compositions

- Capacity for in situ chamber up to $5 \mathrm{~kg}$ and $10 \mathrm{~cm}$ dia.

- Programmable and modular for incorporation of nearly any component for future advancements, replacements, and extended lifetime

- Minimal total consumption of resources

Parameters considered in the evaluation of microfocus $\mathrm{x}$-ray sources included power and voltage range, focal spot size as a function of power, the minimum focus-to-object distance (FOD) which may limit x-ray flux through the sample, target types, and lifetime.

The system magnification (the product of $\mathrm{x}$-ray and detector magnification) can be determined for a given $\mathrm{x}$-ray focal spot size and imaging sensor's pixel size at which neither parameter is limiting the resolution of the system. ${ }^{2}$ The achievable $\mathrm{x}$-ray magnification can be limited by equipment travel range, the size of the $\mathrm{x}$-ray detecting screen and the camera's FOV.

The area of an x-ray producing target (focal spot) limits the resolution of the system at large magnifications. Thin, transmission targets limit spreading of the electron beam within the target, yielding the smallest focal-spot for the highest resolution imaging. Conversely, the reflection-type target configuration can run at much higher power densities yielding a more energetic or brighter beam for denser samples, as discussed by Masschaele et $_{\text {al. }}{ }^{7}$

The selection of x-ray source (X-RAY WorX GmbH XWT-160-SE/TC, Garbsen, Germany) was based on ability to meet the system performance goals. This dual-target tube 


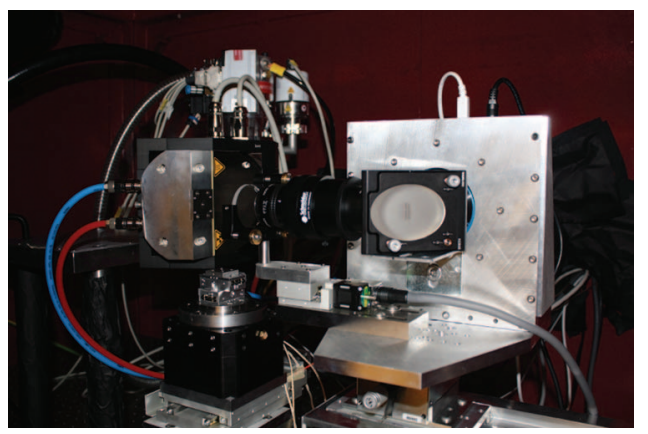

FIG. 1. The constructed X-ray microCT scanner: Shown is the X-ray source (upper left), the sample rotation stage (lower left), and the detector assembly (right).

(transmission/reflection) is capable of $160 \mathrm{kV}$ of accelerating potential yielding high energy $\mathrm{x}$-rays to maximize transmission through large sample and is capable of a very small focal spot size for high resolution imaging. The manufacturer specifies a minimum detail detectability of $<0.3 \mu \mathrm{m}$ and a maximum power of $3 \mathrm{~W}$ with the high resolution transmission target and a minimum detail detectability of $<2.0 \mu \mathrm{m}$ and a maximum power of $280 \mathrm{~W}$ with the reflection target.

Many configurations exist for digitizing sampled x-ray intensities, most relying on scintillating materials to convert $\mathrm{x}$-rays of a given intensity to a proportional amount of optical light, coupled to a light sensitive imaging chip. Phosphors can vary in microstructure from single crystal to columnargrained to powder-compacted, ${ }^{8-11}$ imaging chips are primarily of the CMOS or CCD type, ${ }^{12}$ and the mode of transferring the scintillated light to the light-sensitive detector elements can vary from lens to fiber-optic to direct contact of the phosphor and chip. ${ }^{5,13}$ The imaging system in this design uses a lens-coupled configuration.

The selected camera (Alta U230, Apogee Imaging Systems, Inc., CA) in this system contains a high cosmeticgrade large format $2048 \times 2048$ back-illuminated CCD array (CCD230-42, e2v technologies inc., UK) composed of $15 \mu \mathrm{m}$ square pixels, 16-bit dynamic range, and a peak quantum efficiency of about $95 \%$ at $550 \mathrm{~nm}$ corresponding to the peak emission spectra of some phosphors. Selecting a scintillator with a peak emission wavelength near that of the candidate imaging chip's peak quantum efficiency (QE) improves the detector quantum efficiency (DQE) of the entire detector. A large dynamic range is critical to achieve contrast between phases with similar x-ray attenuation coefficients at the $\mathrm{x}$-ray energy used for imaging. The large-format CCD chip did come with the challenge of identifying a suitable lens for high-resolution imaging which was also able to utilize the entire area the chip. The lens selected for this purpose (MicroSymmar 2.8/50 mm, Schneider Optics, Inc., NY) is a finitefocus macro-lens selected on imaging resolution, a large numeric aperture, low image distortion, and magnification variability.

Selecting the optimum scintillator requires consideration of composition, thickness, emission spectra, density, and light yield for maximizing detection efficiency over a range of $\mathrm{x}$-ray energy balanced against factors such as the thickness, index of refraction and numeric aperture of the lens system (if applicable) to achieve high imaging resolution. ${ }^{11,13}$

Many studies provide great insight into the resolution achievable using lens-coupled single crystal scintillators, showing that thin scintillator screens are necessary in order to achieve the highest imaging resolution. ${ }^{4,9}$ These studies also show that with high NA lenses, higher resolution is possible, but require thinner scintillators to realize. The consequence of a thin scintillator is degraded detector quantum efficiency (DQE) in high-resolution detectors. Using the approach of Koch et al. ${ }^{4}$ the DQE of this system was analyzed as a function of energy for scintillators of varying composition and thickness for the candidate image sensors and couplings. The selection analysis was limited to compositions and dimensions which were commercially available. The higher attenuation of LuAG:Ce at high x-ray energy for a given thickness is preferred for high energy lab-scale radiography, which is not necessarily the case for synchrotron XCT, which use relatively lower energy x-rays.

Given the multi-scale goals of this instrument, an interchangeable scintillator approach was adopted, which is a practical necessity for imaging samples of different sizes and composition. Still, a scintillator composition and thickness was identified to bridge all the performance goals. The scintillator implemented in this system was a single crystal freestanding LuAG:Ce circular disk $250 \mu \mathrm{m}$ thick (Crytur, spol. s r. o., Turnov, Czech Republic) selected based on achievable resolution, the resulting $\mathrm{DQE}$, and commercial availability.

To rotate the specimen in the XCT scan, a stage with perfect rotation without physical distortion and with accurate positioning is ideal. A small footprint and a high load capacity are desirable. In real stages, undesirable procession, tilt, wobble, or run-out may occur during rotation. The rotation stage in this system (ORT-101-L Air Rotary Air Bearing with a Delta Tau MAC-MC-1A-SD Controller, Nelson Air Corp., $\mathrm{NH}$ ) was selected to minimize distortions while providing $27 \mathrm{~kg}$ load capacity, and high precision within $0.00001^{\circ}$ (Fig. 2).

The computed tomography reconstruction workstation utilizes a $3.3 \mathrm{GHz}$ quad-core processor (Intel Corporation, CA) in addition to a 448 core 6 GB CUDA GPU (Tesla c2075, NVIDIA Corporation, CA) for accelerated

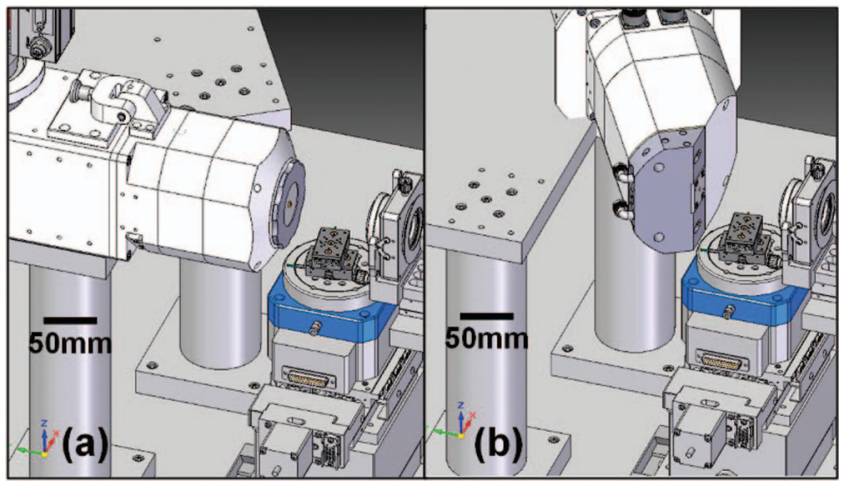

FIG. 2. Computer-aided design model showing the system configuration with both the (a) transmission and (b) reflection x-ray target heads being implemented (left), the sample stack (middle), and the tip of the detector (right). 
reconstruction algorithm execution. RAM on the system is also critical: Approximately twice the projection data size plus 5-10 GB is required to run cone-beam reconstructions without breaking the data up into subsets.

Other components are practical for XCT. Shielding is required around the apparatus to contain X-rays. A vibrationdamping breadboard table top and vibration isolator support columns (RS2000-36-8 \& S-2000A-428, Newport Corporation, CA) were selected as the foundation for the system, based on the installation site. The breadboard top allows for component mounting, and very large $\mathrm{x}$-ray magnifications due to its $6 \mathrm{ft}$. length.

Centering the specimen on the center of the rotation stage is a needed to maximize the FOV. Two linear-axis piezo centering stages of $5 \mathrm{~kg}$ load capacity and $2 \mathrm{~nm}$ resolution (PPS28, Micos USA LLC, CA) were implemented for this purpose (Fig. 2). The high load capacity facilitates large in situ experimentation jigs and the high resolution allows for accurate positioning of the sample being imaged.

Acquisition of flat calibration images is performed with a high-load, long-range motorized linear stage with high repeatability (XA10A-R2, Kohzu Precision, Alio Industries, $\mathrm{CO})$ established orthogonal to the central axis of the $\mathrm{x}$-ray beam, with a $20 \mathrm{~kg}$ load capacity and a $\pm 0.2 \mu \mathrm{m}$ repeatability for this purpose (Fig. 2).

Long-range linear stages (Unislide A6012, Velmex, Inc., NY) were adopted in order to position the sample and detector with the central axis of the x-ray cone and each other selected on the range of magnifications desired and the necessary load capacity.

The selection and integration of components into the design was performed with Computer-Aided Design (CAD) by incorporating commercial part models and custom designs. The dual-target $\mathrm{x}$-ray cradle was designed to maintain beam position and emission axis when switching between $\mathrm{x}$-ray source target heads (Fig. 2). Simulations were performed on the $\mathrm{x}$-ray cradle design to minimize deflection between the positions.

To handle the scintillator, a housing was designed to eliminate dust from settling on the scintillator, which results in dead-zones in the detection system. This was achieved by containment of the scintillator within a sealed optical tube (SM1L10, Thorlabs Inc., NJ) between two retaining rings. The "front" of the tube was sealed off by a 0.001 in. thick layer of Kapton film; the material at the front of the tube attenuates signal $\mathrm{x}$-rays to some extent, which was minimized. The back was sealed by a $6 \mathrm{~mm}$ leaded glass window to pass optical signal and minimize stray $\mathrm{x}$-rays which cause CCD noise. The tube was optically sealed at the front by with a graphite film and in the back by a bellows linking to the lens. The scintillator tube is mounted in a mirror mount (U200A3H, Newport Corporation, CA).

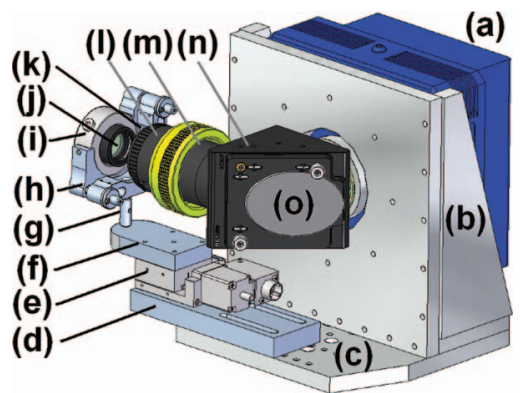

FIG. 3. Detector assembly in CAD software: (a) Camera, (b) Camera support and shield, (c) Base plate, (d) Long-range extension plate, for changing extension tubes, (e) Focusing stage, (f) Mirror-mount-to-focusing-stage adapter, (g) Mirror-mount post, (h) Mirror mount, (i) Shaft collar support for, (j) Optical tube assembly, (k) Bellows material (not shown), (l) Lens, (m) Extension tubes and adjustable length collar, (n) Right-angle mirror housing, (o) Right-angle mirror.

The scintillator tube assembly is contained in the detector assembly (Fig. 3). A linear stage (XA04A-R2, Kohzu Precision, Alio Industries, CO) aids in focusing the optical lens system onto the scintillator, with a $10 \mathrm{~mm}$ travel range a $2 \mu \mathrm{m}$ precision: Longer travel was enabled by a custom slider on which the focus stage was mounted. A $4 \mathrm{~kg}$ load capacity is realized.

The authors acknowledge financial support from the Security and Defense Systems Initiative (SDSI) at Arizona State University and the Semiconductor Research Corporation.

${ }^{1}$ M. J. Flynn, S. M. Hames, D. A. Reimann, and S. J. Wilderman, Nucl. Instrum. Meth. Phys. Res. A 353, 312 (1994).

${ }^{2}$ G. Schena, S. Favretto, L. Santoro, A. Pasini, M. Bettuzzi, F. Casali, and L. Mancini, Int. J. Miner. Proc. 75, 173 (2005)

${ }^{3}$ N. Uhlmann, M. Salamon, F. Sukowski, and V. Voland, Nucl. Instrum. Meth. Phys. Res. A 591, 46 (2008).

${ }^{4}$ A. Koch, C. Raven, P. Spanne, and A. Snigirev, J. Opt. Soc. Am. A 15, 1940 (1998).

${ }^{5}$ K. Uesugi, M. Hoshino, and N. Yagi, J. Synchotron Rad. 18, 217 (2011).

${ }^{6}$ M. Dierick, L. Van Hoorebeke, P. Jacobs, B. Masschaele, J. Vlassenbroeck, V. Cnudde, and Y. De Witte, Nucl. Instrum. Meth. Phys. Res. A 591, 255 (2008).

${ }^{7}$ B. C. Masschaele, V. Cnudde, M. Dierick, P. Jacobs, L. Van Hoorebeke, and J. Vlassenbroeck, Nucl. Instrum. Meth. Phys. Res. A 580, 266 (2007).

${ }^{8}$ D. Packham, M. S. thesis, University of Surrey, Surrey, UK, 2010.

${ }^{9}$ T. Martin and A. Koch, J. Synchotron. Rad. 13, 180 (2006).

${ }^{10}$ K. Sato, Y. Hasegawa, K. Kondo, K. Miyazaki, T. Matsushita, and Y. Amemiya, Rev. Sci. Instrum. 71, 4449 (2000).

${ }^{11}$ J. Touš, K. Blažek, L. Pína, and B. Sopko, Rad. Meas. 42, 925 (2007).

${ }^{12} \mathrm{H}$. Graafsma and T. Martin, "Detectors for synchrotron tomography," Advanced Tomographic Methods in Materials Research and Engineering, edited by J. Banhart (Oxford Scholarship Online, 2008), p. 227.

${ }^{13}$ M. Åslund, E. Fredenberg, M. Telman, and M. Danielsson, Rad. Prot. Dosim. 139, 327 (2010). 